Bull. Austral. Math. Soc.

VOL. 56 (1997) [235-237]

\title{
ON CONJECTURE OF RESNIKOFF AND SALDAÑA
}

\author{
WINFRIED KOHNEN
}

\begin{abstract}
In 1974 Resnikoff and Saldaña made a remarkable conjecture about the growth of the Fourier coefficients of a Siegel cusp form $F$ of arbitrary genus $g \geqslant 1$. In the present note, we point out that this conjecture is at least true on average.
\end{abstract}

\section{INTRODUCTION}

In 1974 Resnikoff and Saldaña [4] made a remarkable conjecture about the growth of the Fourier coefficients of a Siegel cusp form $F$ of arbitrary genus $g \geqslant 1$, which for $g=1$ reduces to the classical Ramanujan-Petersson conjecture proved by Deligne (for the precise statement see below).

Little if any motivation was given. In fact, the authors computed several coefficients of the unique normalised cusp form $\chi_{10}$ of weight 10 and genus 2 and argued that these data supported their conjecture. However, as was proved later in the eighties, the function $\chi_{10}$ is the Saito-Kurokawa lift of the unique normalised cusp form on $S L_{2}(\mathbf{Z})$ of weight 18 [2], and the Fourier coefficients of Saito-Kurokawa lifts on the contrary do not satisfy the conjectured growth condition, see for example [1].

In the present note, we want to point out that the conjecture of Resnikoff and Saldaña is, however, at least true on average. For this one has to use results of Siegel $(g=2)[5]$ and Kitaoka $(g>2)$ [3] on the order of magnitude of the class number of positive definite integral quadratic forms of size $g$ with given determinant. Of course, granting these results and the analytic properties of the Rankin-Dirichlet series of $F$, the arguments are very simple and seem to be well-known from other situations. Nevertheless, because of the lack of motivation found in [4] we think that it might be reasonable to state our observation in this short note.

Received 28 October 1996

This paper was written during the author's stay at Macquarie University, Sydney, Australia. The author would like to thank the School of Mathematics, Macquarie University, for hospitality and financial support.

Copyright Clearance Centre, Inc. Serial-fee code: 0004-9729/97 \$A2.00+0.00. 


\section{The Conjecture}

Conjecture. [4] Let $F$ be a cusp form of weight $k$ and genus $g$ on the full Siegel modular group $\Gamma_{g}$ and let $a(T)$ be its Fourier coefficients ( $T$ a positive definite even integral matrix of size $g$ ). Then one has

$$
a(T) \ll_{\varepsilon, F}(\operatorname{det} T)^{(k / 2)-((g+1) / 4)+\varepsilon} \quad(\varepsilon>0) .
$$

If $g \geqslant 2$ the conjecture so far is not known in a single case. If $g=2$, it is wrong if $k$ is even and $F$ is a Saito-Kurokawa lift of a cusp form of weight $2 k-2$ on $\Gamma_{1}$, as pointed out above. Also, if $8 \mid g$ and $k=1+g / 2$, there are counterexamples due to Freitag, see [1] and below.

\section{Some motivation}

We may suppose $F \neq 0$. Let

$$
D_{F}(s):=\sum_{\{T>0\} / G L_{g}(\mathbf{Z})} \frac{|a(T)|^{2}}{\varepsilon(T)}(\operatorname{det} T)^{-s} \quad(\operatorname{Re}(s) \gg 0)
$$

be the Rankin-Dirichlet series attached to $F$ (summation over a set of representatives of positive definite even integral matrices of size $g$ with respect to the $G L_{g}(\mathbf{Z})$-action $T \mapsto U^{\prime} T U\left(U \in G L_{g}(\mathbf{Z}), U^{\prime}=\right.$ transpose of $\left.U\right) ; \varepsilon(T)=\#\left\{U \in G L_{g}(\mathbf{Z}) \mid U^{\prime} T U=\right.$ $T\})$. Then $D_{F}(s)$ has a meromorphic continuation to $\mathbf{C}$, with first pole occurring at $s=k[1]$. Since $D_{F}(s)$ has non-negative coefficients, by a classical argument due to Landau it follows that $D_{F}(s)$ converges for $\operatorname{Re}(s)>k$. Hence by the well-known formula for the abscissa of convergence of a Dirichlet series we have

$$
\sum_{n \leqslant m}\left(\sum_{\{T>0 \mid \operatorname{det}(T)=n\} / G L_{g}(\mathbf{Z})} \frac{|a(T)|^{2}}{\varepsilon(T)}\right) \ll_{\varepsilon, F} m^{k+\varepsilon} \quad(\varepsilon>0) .
$$

Now by $[\mathbf{5}, \mathbf{3}]$ the class number $\#\{T>0 \mid \operatorname{det} T=n\} / G L_{g}(\mathbf{Z})$ for $n$ large is of the same order of magnitude as $n^{(g-1) / 2+\varepsilon}(\varepsilon>0)$, with constants depending on $\varepsilon$. Hence if we assume that the coefficients $|a(T)|^{2} / \varepsilon(T)$ are "of equal growth", one might hope to be allowed to divide by $n^{(g-1) / 2+1}$ to get the growth of each single term in (2). In other words, one might expect that

$$
\frac{|a(T)|^{2}}{\varepsilon(T)} \ll_{\varepsilon, F} n^{k-((g-1) / 2)-1+\varepsilon} \quad(\varepsilon>0)
$$

would hold. By taking square-roots and observing that $\varepsilon(T)$ (for given $g$ ) is universally bounded by reduction theory, one gets (1). 
REMARK. The examples given by Freitag referred to above show that in some cases the coefficients of $F$ may not be "of equal growth" as assumed above, in fact in a very extreme sense as very many of them are zero. More precisely, let $S$ be a positive definite even integral unimodular matrix of size $g$ (these conditions imply $8 \mid g$ ) and put

$$
\begin{aligned}
F(Z):= & \sum_{G \in \mathbf{Z}^{(g, g)}}(\operatorname{det} G) e^{\pi i \operatorname{tr}\left(G^{\prime} S G \cdot Z\right)} \\
& \left(Z \in \mathbf{H}_{g}=\text { Siegel upper half-space of genus } g\right) .
\end{aligned}
$$

The function $G \mapsto \operatorname{det}(G)$ being a spherical harmonic of degree 1, it follows that $F$ is a cusp form of weight $1+g / 2$. Its Fourier coefficients $a(T)$ are zero unless $\operatorname{det} T$ is a perfect square.

On the other hand, if $S$ has no integral automorphism of determinant -1 , then $F$ is not identically zero ( $a(S)$ is not zero), hence there exist infinitely many non-equivalent $T$ with $a(T) \neq 0$. If $G^{\prime} S G=T$, then $(\operatorname{det} G)^{2}=\operatorname{det} T$, hence $a(T)$ is an integer times $\sqrt{\operatorname{det}(T)}$. Thus $F$ does not satisfy (1) which would predict $a(T) \ll_{\varepsilon, F}(\operatorname{det} T)^{1 / 4+\varepsilon}$.

We would expect that examples like the above (on congruence subgroups of $\Gamma_{g}$ ) can occur only for $k$ small with respect to $g$, but for $g \geqslant 2$ we are not aware of any proof of this. For $g=1$ see [6].

\section{REFERENCES}

[1] S. Böcherer and S. Raghavan, 'On Fourier coefficients of Siegel modular forms', J. Reine Angew. Math. 384 (1988), 80-101.

[2] M. Eichler and D. Zagier, The theory of Jacobi forms, Progr. Math. 55 (Birkhäuser, Boston, 1985).

[3] Y. Kitaoka, 'Two theorems on the class number of positive definite quadratic forms', Nagoya Math. J. 51 (1973), 79-89.

[4] H.L. Resnikoff and R.L. Saldaña, 'Some properties of Fourier coefficients of Eisenstein series of degree two', J. Reine Angew. Math. 265 (1974), 90-109.

[5] C.L. Segel, 'Über die Classenzahl quadratischer Zahlkörper', in Collected Works I, (K. Chandrasekharan and H. Maass, Editors) (Springer-Verlag, Berlin, Heidelberg, New York, 1966).

[6] M.-F. Vignerás, 'Facteurs gamma et équations fonctionelles', in Modular functions of one variable VI, (J.-P. Serre and D. Zagier, Editors), Lecture Notes in Mathematics 627 (Springer-Verlag, Berlin, Heidelberg, New York, 1977), pp. 79-103.

Mathematisches Institut der Universität Heidelberg

Im Neuenheimer Feld 288

69120 Heidelberg

Germany 